\title{
Dynamics of a paramagnetic colloidal particle driven on a magnetic-bubble lattice
}

\author{
Alejandro Soba, ${ }^{1}$ Pietro Tierno, ${ }^{1, *}$ Thomas M. Fischer, ${ }^{2}$ and Francesc Saguès ${ }^{1}$ \\ ${ }^{1}$ Departament de Química Física, Universitat de Barcelona, Martí i Franquès 1, 08028 Barcelona, Spain \\ ${ }^{2}$ Institut für Experimentalphysik V, Universität Bayreuth, Bayreuth, Germany
}

(Received 17 April 2008; published 9 June 2008)

\begin{abstract}
We present a theoretical study of the recently observed dynamical regimes of paramagnetic colloidal particles externally driven above a regular lattice of magnetic bubbles [P. Tierno, T. H. Johansen, and T. M. Fischer, Phys. Rev. Lett. 99, 038303 (2007)]. An external precessing magnetic field alters the potential generated by the surface of the film in such a way to either drive the particle circularly around one bubble, ballistically through the array, or in triangular orbits on the interstitial regions between the bubbles. In the ballistic regime, we observe different trajectories performed by the particles phase locked with the external driving. Superdiffusive motion, which was experimentally found bridging the localized and delocalized dynamics, emerge only by introducing a certain degree of randomness into the bubbles size distribution.
\end{abstract}

DOI: 10.1103/PhysRevE.77.060401

PACS number(s): 82.70.Dd, 85.70.Ge, 05.60.Cd

Transport of particles in periodic pinning potentials is a general phenomenon common to numerous systems in condensed matter. Examples are various and include cold atoms in an optical lattice $[1,2]$, flux flow in superconductors $[3,4]$, biological motors $[5,6]$, macromolecules $[7,8]$, and colloidal particles in optical tweezers $[9,10]$ or above magnetic structured substrates $[11,12]$. Besides the scientific interest, there are also technological possibilities which derive from studying these systems, such as sorting colloidal particles [13], separating DNA chains [14], or designing novel electronic and/or semiconductor devices [15]. To study such a phenomenon, colloidal particles represent a suitable choice due to the availability of methods to generate periodic potentials by using, e.g., holographic optical tweezers or patterned magnetic substrates, and the fact that particle motion can be easily followed in real time. In this context, some of us recently observed different dynamical regimes of paramagnetic colloidal particles externally driven above a hexagonal lattice of magnetic bubbles [16]. The bubbles are cylindrical ferromagnetic domains of uniform magnetization located in a continuous film with reverse magnetization. The size of these domains can increase (or decrease) by application of a magnetic field parallel (opposite) to the bubble magnetization direction. The paramagnetic particles, dispersed in water, were deposited above the bubble array and subjected to a magnetic field precessing around the axis normal to the film. Such field controls the size of the bubbles and, at the same time, drives the particles through the lattice in different dynamical modes depending on its frequency and strength. In particular, for low field strengths and at a fixed frequency, the particles were localized around the bubbles describing circular orbits. Increasing the field reduces the size of the bubbles and the particles were able to escape through the array following a ballistic trajectory. Higher field strengths localized again the particle motion in triangular orbits above larger interstitial regions between the bubbles. Remarkably, superdiffusive trajectories were observed when passing from the localized circular orbits to the ballistic and, again, when

\footnotetext{
*ptierno@ub.edu
}

crossing back from the ballistic regime towards the triangular orbits.

Here we present a theoretical study of such system which reproduces the particle dynamics. First we parametrize the potential generated by a hexagonal array of magnetic bubbles. We solve the overdamped equation of motion for a particle externally driven in such potential when superimposing a precessing magnetic field. Similarly to the experiment, we observe localized (circular or triangular) and delocalized (ballistic) trajectories. In contrast, we obtain superdiffusive motion only when we consider a magnetic disorder in the system in the form of a random distribution of the radius of the bubbles.

We consider a paramagnetic particle of volume $V$ placed above a hexagonal array of magnetic bubbles with radius $R$ and lattice vectors $\boldsymbol{a}_{1} \equiv(a, 0), \boldsymbol{a}_{2} \equiv\left(\frac{1}{2} a, \frac{\sqrt{3}}{2} a\right)$ [see Fig. 1(a)]. When subjected to a magnetic field $\mathbf{H}$, the particle acquires a magnetic moment $\mathbf{m}=V \chi \mathbf{H}$, proportional to the field, where $\chi$ is the effective particle magnetic susceptibility $(\chi=0.17)$. The total magnetic field acting over the particle is $\boldsymbol{H}_{t o t}=\boldsymbol{H}_{l}$ $+\boldsymbol{H}_{e x}$, where $\boldsymbol{H}_{l}$ is the field due to the bubble lattice and $\boldsymbol{H}_{e x}$ the external magnetic field which precesses with an angle $\vartheta$ and a frequency $\Omega$ around the $z$ axis, $\boldsymbol{H}_{e x}$ $\equiv\left(H_{0} \sin \Omega t, H_{0} \cos \Omega t, H_{z}\right)$. To parametrize $\boldsymbol{H}_{l}$ we consider first the magnetic potential $\psi$ above the surface of an isolated cylindrical bubble of thickness $t$ and uniform magnetization $-M_{s}\left(M_{s}\right)$ at $z=0(z=-t)[17]$. The magnetic field generated by this bubble is $\boldsymbol{h}=-\nabla \psi$, and the field due an array of such bubbles is $\boldsymbol{H}_{b}=\sum_{n, m} \boldsymbol{h}_{n, m}$ where $n, m$ cross the entire hexagonal lattice. To obtain $\boldsymbol{H}_{l}$ we use the superposition principle and subtract to $\boldsymbol{H}_{b}$ the contribution due to the opposite magnetized film $\boldsymbol{H}_{f}$ [18]. The knowledge of $\boldsymbol{H}_{t o t}$ allows us to calculate the energy of the paramagnetic particle as $U(\boldsymbol{r}, t)$ $=-\mu \mathbf{m} \cdot \boldsymbol{H}_{t o t} \sim \boldsymbol{H}_{t o t}^{2}$, where $\mu$ is the magnetic susceptibility of the medium (water). Figure 1(b) shows the energy contour plot calculated at an elevation $z=1.5 \mu \mathrm{m}$ (from the particle center) with the experimental parameters: $a=11 \mu \mathrm{m}, R$ $=3.4 \mu \mathrm{m}, M_{s}=1.7 \times 10^{5} \mathrm{~A} / \mathrm{m}, H_{z}=0.29 M_{s}$ and $H_{0}=0.1 M_{s}$. The main features to notice are the cylindrical hills centered at the position of the bubbles (black-red regions) and the energy minima around them. Application of a magnetic field 

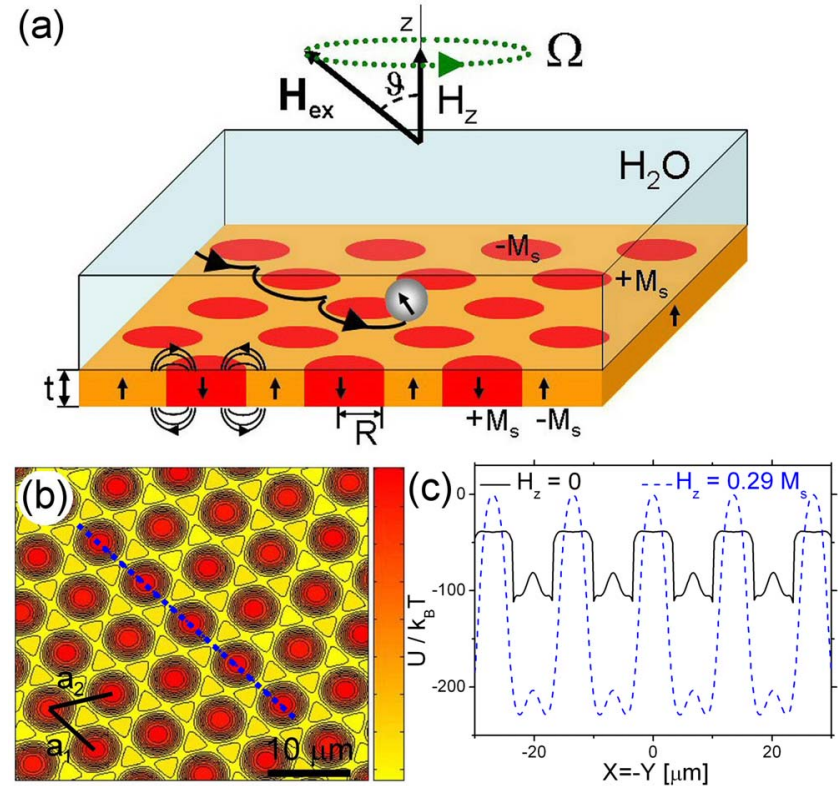

FIG. 1. (Color online) (a) Schematic of a lattice of magnetic bubbles (radius $R$ ) with a paramagnetic particle subjected to a magnetic field $\boldsymbol{H}_{e x}$ precessing with frequency $\Omega$ and angle $\vartheta$ around the $z$ axis. (b) Contour plot of the magnetic potential generated at height $z=1.5 \mu \mathrm{m}$ above the surface $(z=0)$, of the bubble array (lattice vectors $\left.\boldsymbol{a}_{1}, \boldsymbol{a}_{2}\right)$ with $H_{z}=0.29 M_{s}, H_{0}=0.1 M_{s}$. Energy minima correspond to gray (yellow) regions and maxima to black (red) regions. (c) Magnetic potential along the (dashed line) cut in the phase diagram for $H_{z}=0$ (black) and $H_{z}=0.29 M_{s}$ (blue).

normal to the film $H_{z}$ but antiparallel to the magnetization of the bubbles, enlarges the interstitial regions making the energy minima closer and the hills higher. This is illustrated in Fig. 1(c), where we show a cut along the energy landscape for two fields: $H_{z}=0 \mathrm{~A} / \mathrm{m}$ (continuous) and $H_{z}=0.29 M_{s}$ (dashed line). Application of $H_{z}$ also alters the lattice constant $a$ in the film due to magnetic repulsive interactions between the bubbles [19]. To consider this effect we assume a linear dependence of $a$ on $H_{z}$ [20].

The particle trajectory results from the interplay between the externally modulated energy landscape and the viscous friction of the medium. Thus we use an overdamped equation of motion,

$$
\boldsymbol{\nabla} U(\boldsymbol{r}, t)=-f \eta b \dot{\boldsymbol{r}},
$$

where $f$ is the hydrodynamic drag coefficient $(f=27$ [21]), $b$ the particle radius $(b=1.4 \mu \mathrm{m})$ and $\eta$ the water viscosity. We initially $(t=0)$ place a particle at the center of a hexagonal array of typically $64 \times 64$ bubbles, and at elevation $z=1.1 b$, and solve this equation with a time interval $d t=1.4 \times 10^{-4} \mathrm{~s}$ and a total of $\sim 10^{5}$ time steps to ensure a steady condition [22]. The obtained trajectories are analyzed in terms of the correlation function $C(\tau)=\left\langle[\boldsymbol{r}(t)-\boldsymbol{r}(t+\tau)]^{2}\right\rangle_{t} \sim \tau^{\alpha}$ with $\mathbf{r}(t)$ the two-dimensional particle position at time $t$ and where $\langle\cdot\rangle_{t}$ denotes a time average. Depending on the exponent $\alpha$, we classify the particle motion as localized $(\alpha=0)$, ballistic $(\alpha=2)$, or superdiffusive $(\alpha>1)$.

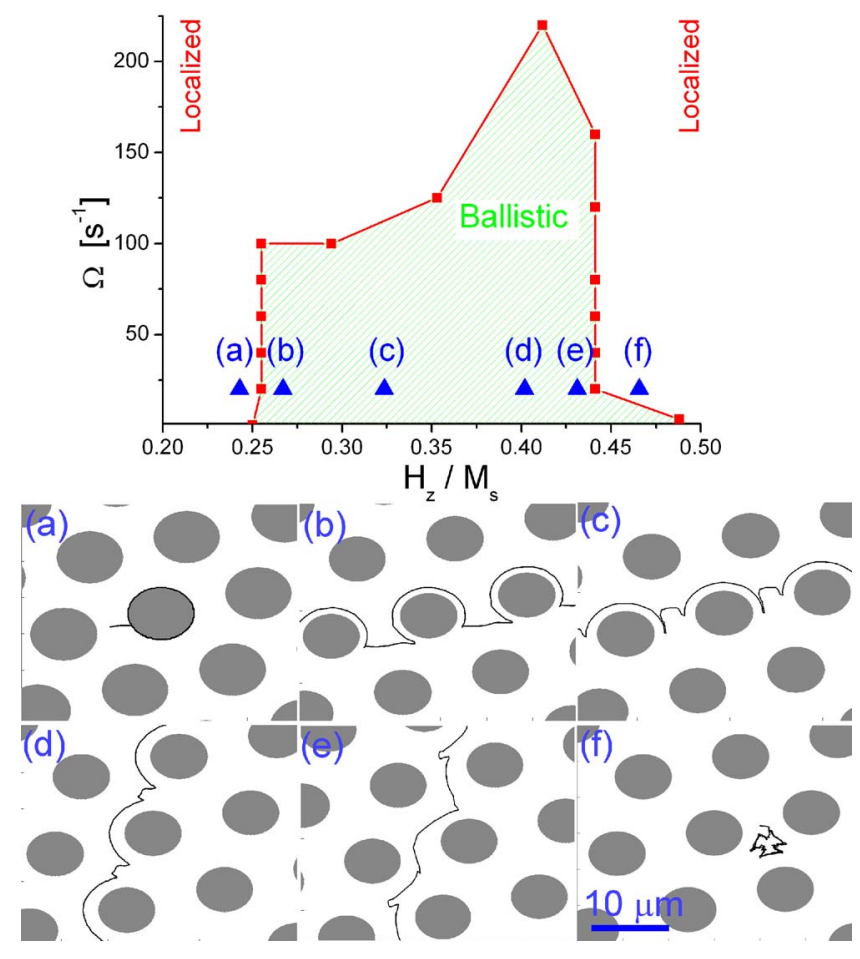

FIG. 2. (Color online) (Top) Phase diagram in the $\left(\Omega, H_{z} / M_{s}\right)$ plane of the exponent $\alpha$. White regions denote localized states $(\alpha=0)$, gray (green) regions correspond to ballistic motion $(\alpha=2)$. (Bottom) The particle trajectories of the localized [(a),(f)] and delocalized states [from (b) to (e)] are shown in the bottom. The blue points in the diagram are the corresponding locations of the bottom panels.

In Fig. 2 we show the phase diagram of the exponent $\alpha$ computed by varying $H_{z}$ and $\Omega$, while keeping the plane component of the applied field $H_{0}$ fixed. We note that, for the field strengths considered here $\left(H_{z}<M_{s}\right)$, only $H_{z}$ controls the size of the bubbles. Representative images of the particle trajectories encountered are depicted at the bottom of Fig. 2. For small field intensities, $0<H_{z}<0.25 M_{s}$, the dynamics are localized and the particle orbits around the bubbles [Fig. 2(a), inset, bottom], as observed in the experiments. The particle follows the planar component $H_{0}$ which results in a synchronous rotation with the field frequency. Further increasing $H_{z}$ decreases the size of the bubbles and widens the energy minima around them which now become closer. For $H_{z} \geq 0.25 M_{s}$ the dynamics becomes delocalized and the particle settles into a stable ballistic trajectory [Fig. 2(b), inset, bottom]. In this case, $H_{0}$ breaks the symmetry of the potential and the particle instead of circulating around the entire bubble passes to a consecutive one where it finds the closer energy minimum. The particle trajectory could be directed along any of the six crystallographic axis of the array, depending on the initial position of the particle relative to the orientation of $H_{0}$. We also observe similarly to [16] that the ballistic region in the phase diagram closes at frequencies higher than $\sim 200 \mathrm{~s}^{-1}$, when the damped particle cannot follow the fast field dynamics and it localizes again. This feature together, with the reproduced phase diagram confirm that our model describes the experimental results. In our simulations we also find that the ballistic regime closes be- 
low $\Omega<10^{-3} \mathrm{~s}^{-1}$. It is hence clear that the lagging of the particle behind the magnetic energy minima is an essential feature for the occurrence of ballistic motion. The experiments of Ref. [16] were all carried out well above this threshold.

Two striking results are worth mentioning at this point. First, we distinguish up to three ballistic trajectories in the delocalized region, $0.32 M_{s}<H_{z}<0.44 M_{s}$, which were not previously observed in the experiments. For $H_{z}>0.32 M_{s}$, the ballistic path changes and the particle after jumping to a consecutive bubble moves first forward then backward along the circular domains and further passes to the next bubble [see Fig. 2(c), bottom inset]. Further increasing $H_{z}$ enlarges the part of the path which passes through the interstitial regions and correspondingly shortens the transit time of the particle around the bubbles [Figs. 2(d) and 2(e) bottom insets]. The numerical method employed allows us to resolve such different paths something which was not possible with the experimental resolution. When $H_{z}$ increases above $0.44 M_{s}$ the trajectory becomes again localized and the particle performs triangular orbits on the interstitial regions between the bubbles. Here the strength of $H_{z}$ is such that the energy minima between three nearest bubbles are interconnected and trap the particle in a closed orbit.

The second remarkable result is the absence of a superdiffusive regime that bridges the localized and ballistic motions. Indeed in the phase diagram of Ref. [16] we observed two superdiffusive regions, respectively, on each side of the ballistic region, spanning an area of $3 \times 10^{-2} M_{s}$. We confirm the lacking of superdiffusion in our model by deeply exploring the transition lines between the two dynamical phases up to a precision of $10^{-4} M_{s}$. The fact that these regimes were present in the experiments at the transitions between stable dynamical phases suggests the presence of some degree of noise in the system which could destabilize the dynamics during transition. We first conjectured that this effect could arise from thermal fluctuations that were not included initially in our model. However, by adding a thermal noise to Eq. (1) and solving this equation at different temperatures, we did not observe any change in the particle trajectories at the transition lines. Actually the large magnetic strengths by which the magnetic potential pins the particles $(\sim 100 \mathrm{pN})$ compared to the Brownian forces $(\sim f \mathrm{~N})$ explains the negligible role played by the thermal noise.

Another possibility is to consider a magnetic disorder in the film which arises during application of the field. Indeed the planar component of the rotating field $H_{0}$, while unable to change the volume of the bubbles, could induce very small radial deformations of the magnetic domains [23], unobservable with the microscope system used in [16]. One computationally efficient way which we found was to add some degree of randomness to the distribution of the radii of the bubbles and periodically refresh it with a time constant $\tau_{\text {ref }}$. Figure 3 shows a superdiffusive trajectory at the transition line from a circularly localized to a ballistic trajectory $\left(H_{z}\right.$ $=0.25 M_{s}, \Omega=20 \mathrm{~s}^{-1}$ ) by using a uniform random distribution of the radii of the bubbles, between $R+\sigma R$ and $R-\sigma R$ with $\sigma=0.1$ and $\tau_{r e f}=0.02 \mathrm{~s}$. Computing $C(\tau)$ we determined a superdiffusive exponent $\alpha=1.4$. in the long-time limit of our simulation. It should be emphasized that this behavior occurs

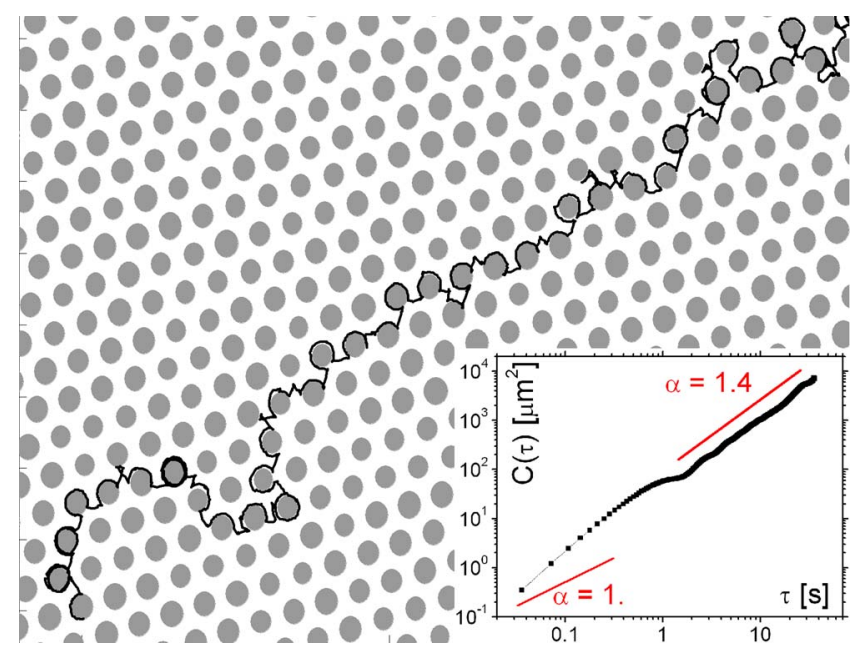

FIG. 3. (Color online) Superdiffusive trajectory of a paramagnetic particle subjected to a field with $H_{z}=0.25 M_{s}, H_{0}=0.1 M_{s}$, and $\Omega=20 \mathrm{~s}^{-1}$. The radii of the bubbles were periodically varied every $0.02 \mathrm{~s}$ (see text). Lower inset shows the correlation function $C(\tau)$ with a superdiffusive exponent $\alpha=1.4$.

only close to the transition point. Numerical simulation with the same value of $\sigma$ inside either of the two localized or the ballistic regions do not show any feature of superdiffusive behavior.

The superdiffusive dynamics was observed by varying periodically in time the radii of the bubbles in the film. This means that this particular regime can be characterized in terms of two parameters, the radius dispersion $\sigma$ and the refreshing time $\tau_{\text {ref }}$. We performed a series of numerical integrations close to the two transition points at fixed frequency, $\Omega=20 \mathrm{~s}^{-1}$. The results are shown in Fig. 4 in terms of the exponent $\alpha$, where graph 4(a) was obtained for $H_{z}$ $=0.23 M_{s}$ (transition from circular localized to ballistic) and graph 4(b) for $H_{z}=0.46 M_{s}$ (transition from ballistic to triangular localized). Both graphs present the same trend: for low and high refreshing times the dynamics is still localized ( $\alpha$ $=0$ ), being superdiffusive only for intermediate values of $\tau_{\text {ref. }}$. This can be explained by considering the behavior of a system subjected to a periodic forcing. When the forcing is too fast $\left(\tau_{r e f} \longrightarrow 0\right)$, the particle does not feel the fast vibrations of the bubbles and its trajectory remains unperturbed with respect to the situation in absence of disorder. The same
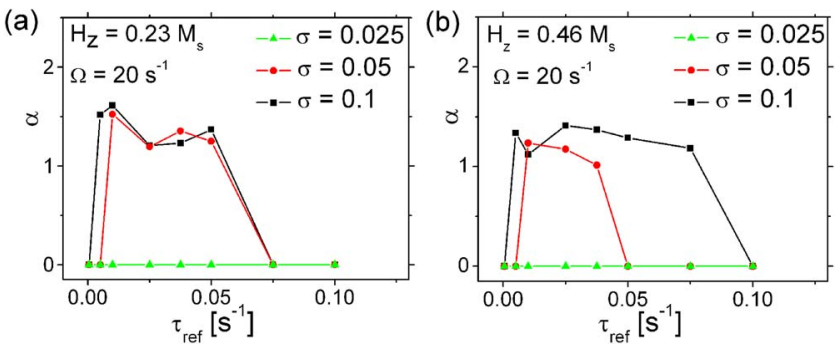

FIG. 4. (Color online) $\alpha$ versus $\tau_{\text {ref }}$ for $\sigma=0.025,0.05$, and 0.1 taken at the threshold between the circularly localized and the ballistic trajectory (a) and between the ballistic trajectory and triangular orbits (b). 
situation happens when the polydispersity of the bubble is frozen $\left(\tau_{r e f} \rightarrow \infty\right)$ since the motion in this case is permanently localized without the freedom to explore the changing landscape.

Transition from localized to delocalized dynamics above periodic pinning potentials has been observed [24] and theoretical described [25] in the magnetotransport of electrons through an array of scatterers, in the so-called "electron pinball." Also theoretical work has been done in the context of vortex motion in a pinning potential [26] and particle transport trough periodic substrates [27]. Of the cited manuscripts, our simulation shows similar features with Ref. [26] under the condition of zero dc drive, where transport into a defined direction is obtained via symmetry breaking, since the system has no preferred direction of motion. Also ballistic particle motion phase locked with the external field for an overdamped driven system was shown in Ref. [27]. Where our work differs, apart from the physical system to which it refers, is in the implementation of some degree of disorder in the pinning potential which allows one to obtain the experimentally observed superdiffusive particle trajectories.

In summary, we theoretically studied the motion of paramagnetic colloidal particles externally driven above a magnetic bubble lattice. We explored the particles dynamics in a wide range of fields and frequencies and obtain, in most of the cases, satisfactory agreement with the experimental data.
Where our finding differs is in the various ballistic trajectories of the particles and in the absence of a superdiffusive regime. We attributed the latter to dynamical disorder in the magnetic potential. Implementing this hypothesis in our model in terms of a time changing random distribution in the effective radii of the bubbles allows one to capture the superdiffusive trajectories. The simple model described in this Rapid Communication can be extended in various ways. For example one can study the motion of particles with different size and/or magnetic content and observe different dynamics (coexistence of localized and delocalized states) useful in sorting and fractionating colloidal particles. Also plans to investigate motion of the particles above magnetic lattices with frozen defects, different geometry of the magnetic domains, or lattice morphologies (such as square, oblique, etc.) are on the way.

The authors benefit from stimulating discussions with Jose M. Sancho and Ana M. Lacasta. A.S. acknowledges the AECI Program, Project A/8402/07. P.T. was supported by Beatriu de Pinós Program No. BP-B100167. F.S. and P.T. acknowledge financial support by MEC (Project No. FIS2006-03525) and DURSI (Contract No. 2005SGR00653), T.M.F. by the National Science Foundation under Contract No. CHE-0649427.
[1] E. Lundh and M. Wallin, Phys. Rev. Lett. 94, 110603 (2005).

[2] C. Mennerat-Robilliard, D. Lucas, S. Guibal, J. Tabosa, C. Jurczak, J. Y. Courtois, and G. Grynberg, Phys. Rev. Lett. 82, 851 (1999).

[3] C. Reichhardt and F. Nori, Phys. Rev. Lett. 82, 414 (1999).

[4] K. Kilic, A. Kilic, H. Yetis, and O. Cetin, Phys. Rev. B 68, 144513 (2003).

[5] K. Svoboda et al., Nature (London) 365, 721 (1993).

[6] F. Jülicher, A. Ajdari, and J. Prost, Rev. Mod. Phys. 69, 1269 (1997).

[7] J. Fu, P. Mao, and J. Han, Appl. Phys. Lett. 87, 263902 (2005).

[8] N. Laachi, C. Declet, C. Matson, and K. D. Dorfman, Phys. Rev. Lett. 98, 098106 (2007).

[9] P. T. Korda, M. B. Taylor, and D. G. Grier, Phys. Rev. Lett. 89, 128301 (2002).

[10] K. Mangold, P. Leiderer, and C. Bechinger, Phys. Rev. Lett. 90, 158302 (2003).

[11] B. B. Yellen, O. Hovorka, and G. Friedman, Proc. Natl. Acad. Sci. U.S.A. 102, 8860 (2005).

[12] P. Tierno, S. V. Reddy, T. H. Johansen, and T. M. Fischer, Phys. Rev. E 75, 041404 (2007).

[13] A. M. Lacasta, J. M. Sancho, A. H. Romero, and K. Lindenberg, Phys. Rev. Lett. 94, 160601 (2005).

[14] L. R. Huang et al., Anal. Chem. 75, 6963 (2003).

[15] U. K. Mishra and J. Singh, Semiconductor Device Physics and Design (Springer Netherlands, Amsterdam, 2007).
[16] P. Tierno, T. H. Johansen, and T. M. Fischer, Phys. Rev. Lett. 99, 038303 (2007).

[17] W. F. Druyvesteyn, D. L. A. Tjaden, and J. W. F. Dorleijn, Philips Res. Rep. 27, 7 (1972).

[18] For symmetry reasons we estimate the contribution of the magnetic film, $\boldsymbol{H}_{f}$, by considering a cylindrical domain covering the entire bubble area. The total magnetic field is thus $\boldsymbol{H}_{t o t}=\boldsymbol{H}_{e x}+\boldsymbol{H}_{b}-\boldsymbol{H}_{f}$.

[19] R. Seshadri and R. M. Westervelt, Phys. Rev. B 46, 5150 (1992).

[20] $a / D=4.1-2\left(H_{z} / M_{s}\right)$, where $D=3.4 \mu \mathrm{m}$.

[21] P. Tierno, R. Muruganathan, and T. M. Fischer, Phys. Rev. Lett. 98, 028301 (2007).

[22] To reach computational speed, we calculate exactly the magnetic contribution due to all bubbles surrounding the particle and contained in a disk of radius $d=3 R$. The other bubbles are approximated as point dipoles of strength $2 M_{s} \pi R^{2} t$. The goodness of this approximation is discussed in Ref. [17].

[23] J. C. Slonczewski, B. E. Argyle, and J. H. Spreen, IEEE Trans. Magn. 17, 2760 (1981).

[24] D. Weiss et al., Phys. Rev. Lett. 66, 2790 (1991).

[25] R. Fleischmann, T. Geisel, and R. Ketzmerick, Phys. Rev. Lett. 68, 1367 (1992)

[26] C. Reichhardt and C. J. Olson, Phys. Rev. B 65, 100501(R) (2002).

[27] C. Reichhardt and C. J. Olson, Phys. Rev. E 68, 046102 (2003). 